\title{
Der Bronchoskopiestuhl
}

\section{An Aid to Bronchoscopy and Local Endobronchial Anaesthesia: the Bronchoscopy Seat}

Autoren

Institut

\section{R. Kropp, R. Dierkesmann}

Das Deutsche Tuberkulose-Archiv e. V., Petersberg

\section{Bibliografie}

DOI $10.1055 / s-2008-1038286$

Online-Publikation: 29.10.2008

Pneumologie 2009; 63: 404-406

(C) Georg Thieme Verlag KG

Stuttgart · New York

ISSN 0934-8387

Korrespondenzadresse

Dr. med. Robert Kropp

Das Deutsche Tuberkulose-

Archiv e. V.

Liegnitzer Straße 5

36100 Petersberg

dr.robert.kropp@gmx.de

Serienherausgeber

R. Kropp, Fulda

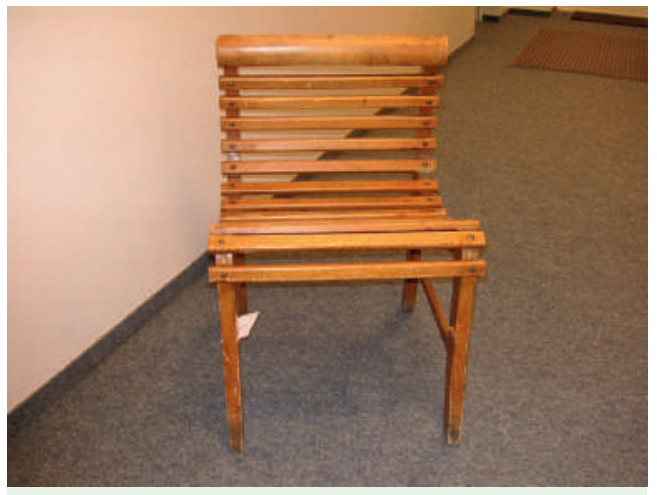

Abb. 1 Bronchoskopiestuhl von vorn.

Vor einiger Zeit wurde dem Deutschen Tuberkulose-Archiv ein eigentümliches, kleines Stühlchen geschenkt, welches zunächst unbekannt und dessen Bedeutung völlig unklar war. Nachforschungen blieben lange Zeit ergebnislos, führten aber letztendlich doch zum Ziel: Es ist ein Bronchoskopiestuhl! ( $\bullet$ Abb.1).

„Unter direkter Tracheobronchoskopie versteht man die Besichtigung des Tracheo-Bronchialbaumes mithilfe starrer Rohre, die entweder vom Munde her durch den Kehlkopf hindurch (obere Tracheobronchoskopie) oder durch eine Tracheotomieöffnung (untere Tracheobronchoskopie) eingeführt werden können. Der oberen Methode geht stets eine direkte Besichtigung des Kehlkopfes (direkte Laryngoskopie oder Autoskopie) voraus“ [1]. Die Tracheobronchoskopie, die von Gustav Killian 1897 entwickelt worden war ${ }^{1}$, wurde rasch bekannt, weithin angewandt und technisch verbessert, vor allem hinsichtlich der Lichtführung und des Instrumentariums. Die Untersuchung erfolgte mit starrem Rohr vorzugsweise in Lokalanästhesie der Schleimhaut mit 10\% Kokainlösung durch Pinselung, Wattetupfer oder Spray. Diese Lokalanästhesie war eingeschränkt durch die kurze Wirkungszeit des Kokain und dessen Toxizität, das Mittel konnte des- wegen nicht ad libitum verwandt werden. Die Untersuchung musste also zügig erfolgen.

Die Einführung des starren Bronchoskopes in die Trachea war je nach Körperbau des Patienten mit erheblichen Schwierigkeiten verbunden, musste doch zwischen Zahnreihe und Kehlkopfeingang eine gerade Linie hergestellt werden, um das Instrument in die Trachea vorbringen zu können. Bis Mitte des 19. Jahrhunderts hielt man eine solche direkte Betrachtung der Trachea anatomisch nicht für möglich: Ein Mr. Green, der 1846 auf einem Kongress über eine solche Endoskopie berichtet hatte, wurde kurzer Hand der Lüge bezichtigt und aus der wissenschaftlichen Gesellschaft ausgeschlossen [2]. Erst Ende des 19.Jahrhunderts hat Gustav Killian dieser Methode zum Durchbruch verholfen, nachdem er an der Leiche die direkte Zugangsmöglichkeit in die Trachea eindeutig nachgewiesen hatte.

Die Untersuchung wurde selten im Liegen mit hängendem Kopf, vorwiegend im Sitzen vorgenommen. Letzteres wurde als günstiger angesehen, weil der Patient beweglicher war und sozusagen „um das starre Bronchoskopierohr herum“ bewegt, gebogen werden konnte, um Einblick in die einzelnen Bronchien zu erlangen.

Für die Endoskopie im Sitzen benötigte man einen niedrigen Schemel oder einen von Brüngings [3] entwickelten, besonders geeigneten Untersuchungsstuhl ( $\bullet$ Abb.2), der durch seine Höhe sowie die Form des Sitzes und der Rückenlehne den Patienten zur „richtigen“ Haltung veranlasste. Der Patient nahm auf ihm Platz und beugte seinen Oberkörper stark nach vorn, indem er seinen Hals möglichst weit nach vorn reckte. Dann fasste er mithilfe eines kleinen Tuches seine Zunge

\footnotetext{
${ }^{1}$ Einzelheiten, vor allem auch die genaue Beschreibung der Untersuchungsmethode, können im Nachdruck der ursprünglichen Killianschen Publikation nachgelesen werden („Historisches Kaleidoskop“, Pneumologie 2005; 59: 725-729).
} 


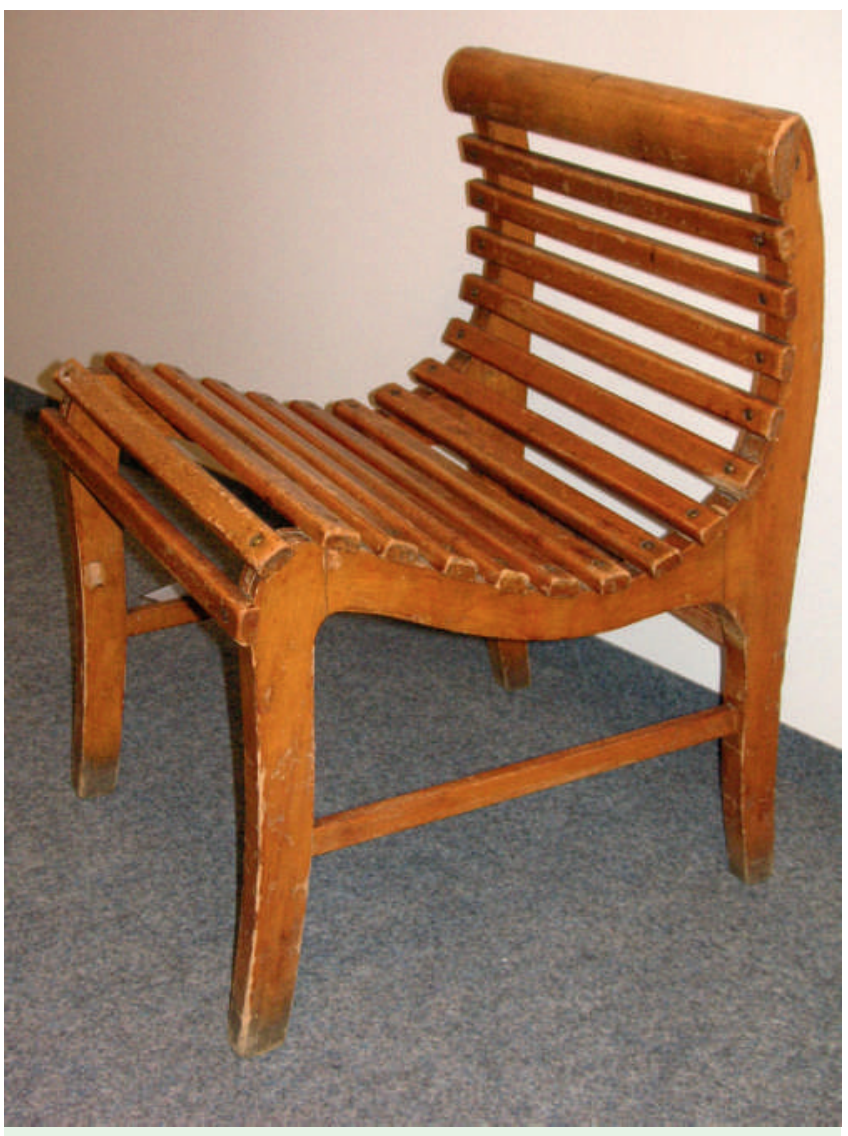

Abb.2 Bronchoskopiestuhl.

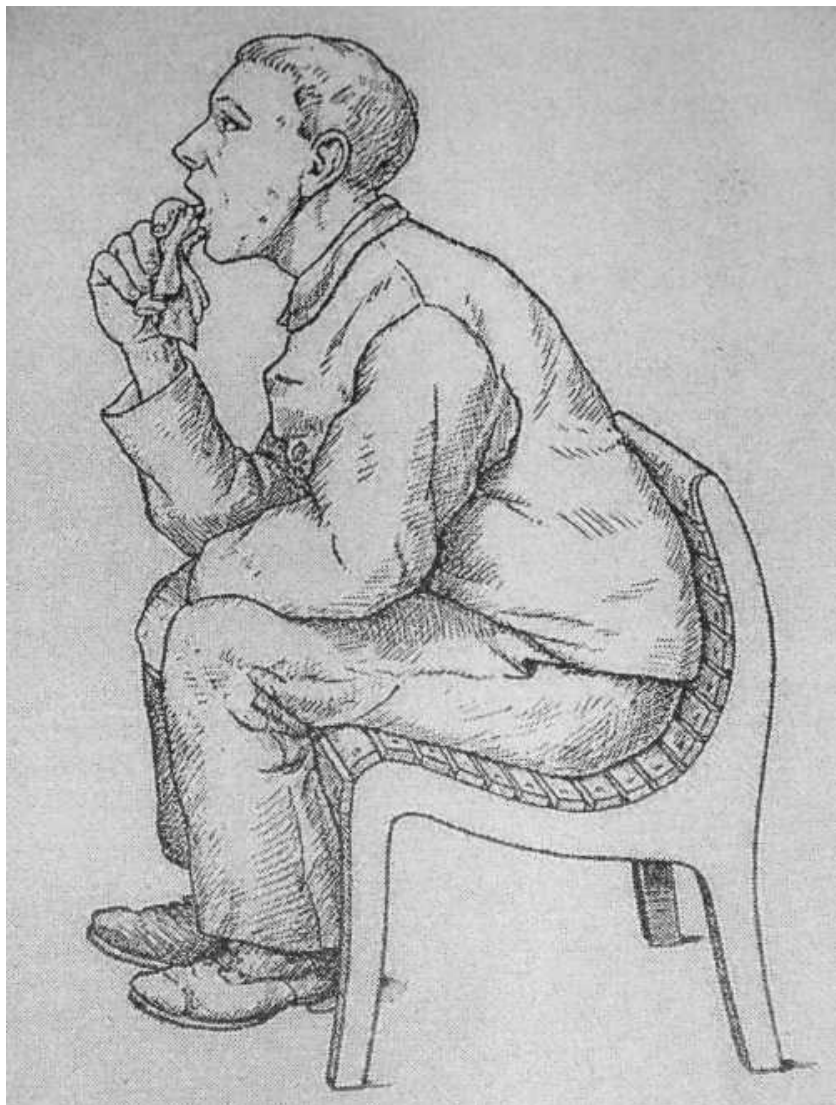

Abb.3 Sitzhaltung.

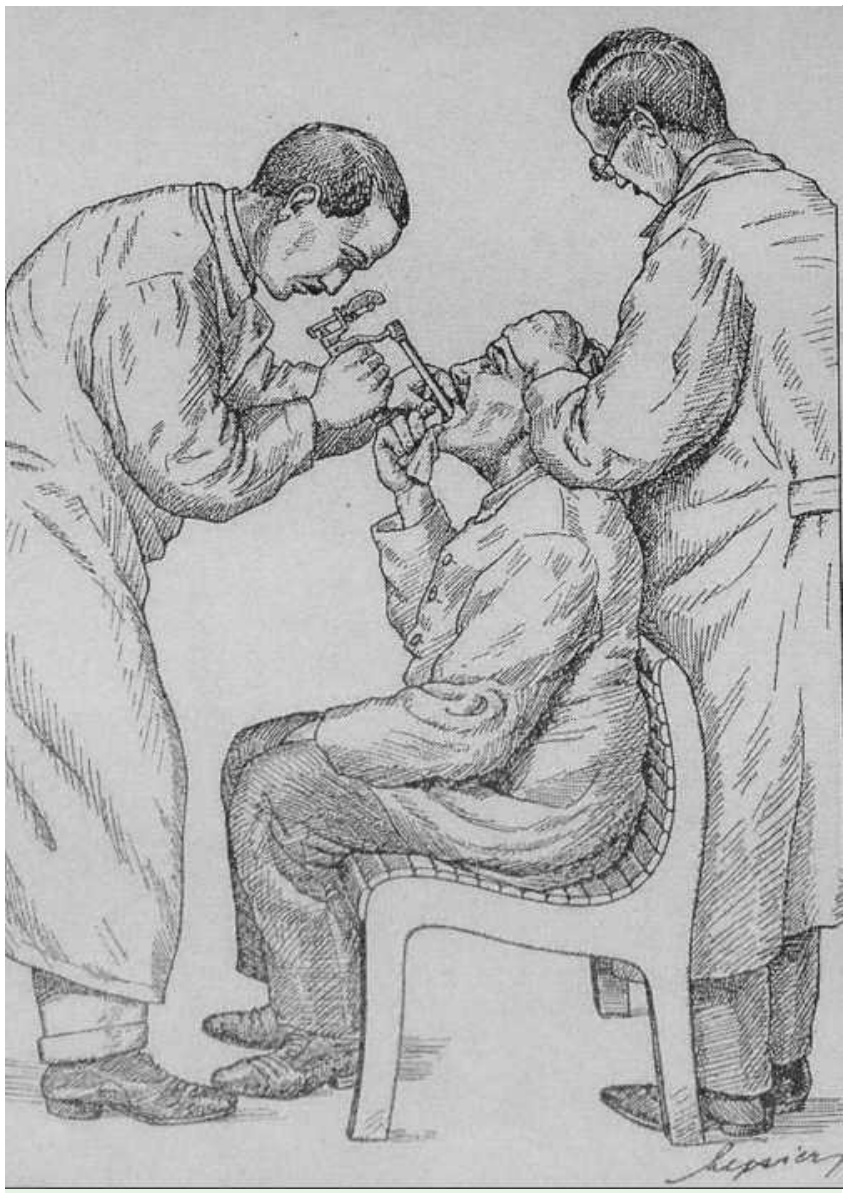

Abb.4 Einführen des Endoskopierohres.

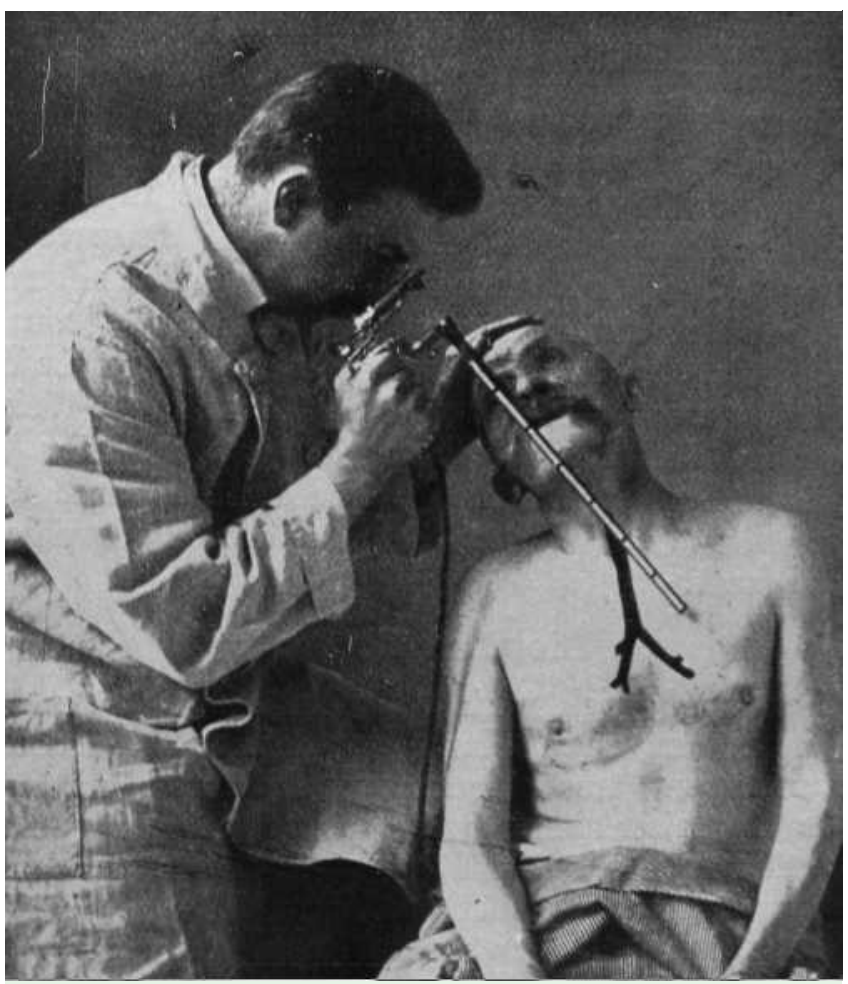

Abb.5 Darstellung des linken Hauptbronchus. 


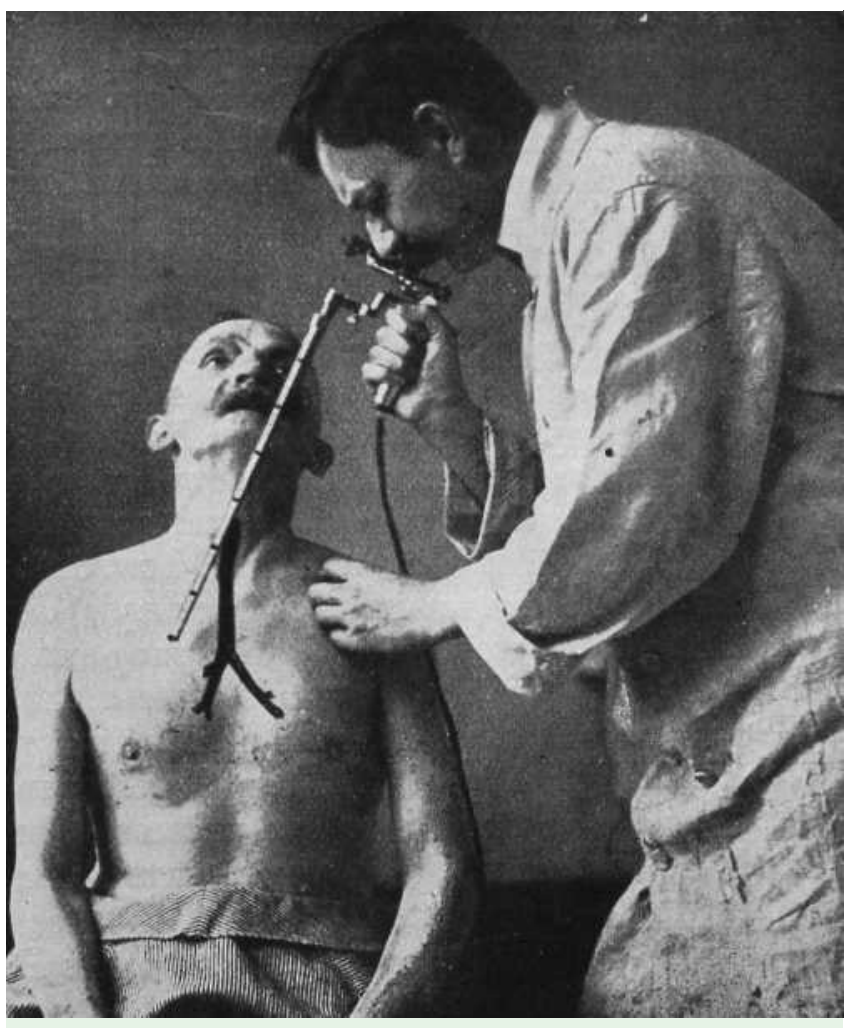

Abb.6 Darstellung des rechten Hauptbronchus.

und zog sie mit der rechten Hand so weit als möglich vor (৫ Abb.3).

Der Arzt hielt mit seiner rechten Hand das Instrument, mit der linken hielt er Oberlippe und Bart des Patienten zurück und gab damit zugleich dem einzuführenden Rohr ein sicheres Gleitlager. Dann wurde das Bronchoskop genau in der Medianlinie unter Niederdrücken der Zunge vorgeschoben, bis unter die Epiglottis. Mit einer hebelnden Bewegung des Instrumentes wurde die Arygegend ins Blickfeld gebracht. Nach Loslassen der Zunge wurden durch kräftigen Druck auf den Zungengrund die Stimmbänder eingestellt, wobei beachtet werden musste, dass die Oberzähne vom direkten Druck des Rohres verschont blieben. Eventuell durch Änderung der Oberkörperbeugung wurde erreicht, dass die Trachea die direkte Verlängerung des Bronchoskopes bildete ( $\mathbf{A}$ bb.4). Mit langsam tiefer gleitenden und rotierenden Bewegungen erfolgte dann die Kehlkopfpassage, während der Patient ruhig atmete (nach [1]). Die weiteren Einstellungen des Instrumentes gelingen nicht allein durch dessen beschränkte Bewegungsmöglichkeiten, sondern durch gleich- zeitige „Modellierung des Patienten“ [3], nämlich durch lordotische, kyphotische und skoliotische Verbiegungen der Hals- und Brustwirbelsäule ( $\bullet$ Abb.5 u. $\bullet 6)$.

Diese Art der Bronchoskopie ohne Vollnarkose mutet uns heute heroisch an und der Bronchoskopiestuhl erscheint uns eher als ein Folterstuhl. Allerdings muss man sich in Erinnerung rufen, dass die versehentliche Aspiration eines Fremdkörpers für den Betroffenen in der damaligen Zeit häufig eine lebenslange Plage war; in Vergleich dazu war die vorübergehende Tortur auf einem solchen Stuhl das geringere Übel. Auf jeden Fall muss den Pionieren der Bronchoskopie hoher Dank gezollt werden; ohne deren Vorarbeit wäre es wahrscheinlich nicht zu dieser großartigen Verfeinerung der Technik gekommen, die uns heute eine fast mühelose und für den Patienten in der Regel gut verträgliche Inspektion und sogar auch Manipulation im Bronchialsystem erlaubt $($ Abb. 7).

Die Abbildungen sind den beiden Literaturstellen entnommen. Die Fotografien stammen aus dem Deutschen TuberkuloseArchiv.

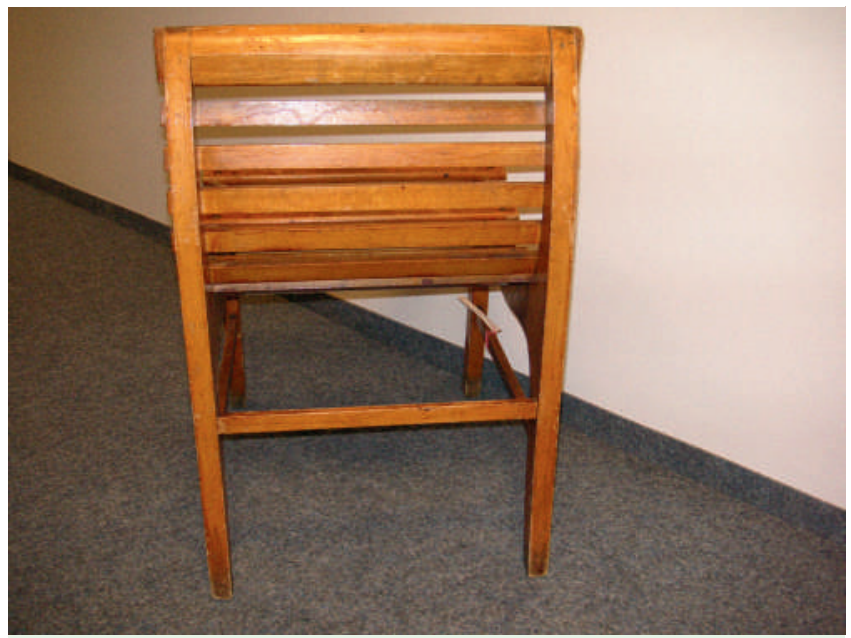

Abb. 7 Bronchoskopiestuhl von hinten.

\section{Literatur}

1 Frenzel H. Die Tracheo-Bronchoskopie. In: Kirschner M, Nordmann O (Hrsg). Die Chirurgie. Eine zusammenfassende Darstellung der allgemeinen und der Speziellen Chirurgie. Band IV, 2. Teil. Berlin: Urban \& Schwarzenberg, 1928: $383-385$

2 Marsh BR. Historic development of bronchoesophagology. Otolaryngology - Head and Neck Surgery 1996; 6: 689-716

3 Brünings $W$, Albrecht $W$. Direkte Endoskopie der Luft- und Speisewege. Neue Deutsche Chirurgie, Band 16. Stuttgart: Enke, 1915 